\title{
Application of biomarkers in the diagnosis of uncertain samples of core needle biopsy of thyroid nodules
}

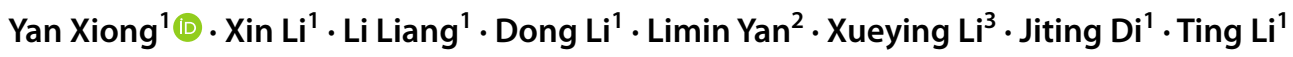

Received: 9 February 2021 / Revised: 10 July 2021 / Accepted: 12 July 2021 / Published online: 26 July 2021

(c) The Author(s) 2021

\begin{abstract}
Core needle biopsy $(\mathrm{CNB})$ is now more frequently used for the preoperative diagnosis of thyroid nodules. Based on morphology alone, 5-20\% of CNB samples cannot be determined as malignant or benign. Compared to fine-needle biopsy (FNB), samples collected by CNB are more accessible for various tests. Therefore, studying biomarkers' application in distinguishing uncertain CNB samples of thyroid nodules is a practical need. Patients of thyroid nodules with both CNB and matched resected specimens were reviewed. Cases classified as indeterminate lesions, follicular neoplasms, and suspicious for malignancy were retrieved. All CNB samples were stained by immunohistochemistry (IHC) using antibodies against CK19, galectin-3, HBME-1, and CD56 and detected by next-generation sequencing (NGS) using an OncoAim® thyroid cancer multigene assay kit (Singlera Genomics) that detected 26 genes. Taking the resected specimens' classification as the gold standard, the sensitivity, specificity, positive predictive value (PPV), negative predictive value (NPV), accuracy of a single biomarker, and various combinations for discriminating malignancy from benignity were calculated. The sensitivity, specificity, PPV, NPV, and accuracy for preoperative malignancy evaluation were as follows. In the cohort of non-follicularneoplasm-lesions (non-FN-lesion), they were $95.16 \%, 53.85 \%, 90.77 \%, 70.00 \%$, and $88.00 \%$ for CK19; 95.16\%, 38.46\%, $88.06 \%, 62.50 \%$, and $85.33 \%$ for galectin- $3 ; 77.42 \%, 76.92 \%, 94.12 \%, 41.67 \%$, and $58.00 \%$ for HBME- $1 ; 66.13 \%, 100.00 \%$, $100.00 \%, 38.24 \%$, and $72.00 \%$ for CD56; $90.32 \%, 92.31 \%, 98.25 \%, 66.67 \%$, and $90.67 \%$ for NGS; and $88.71 \%, 92.30 \%$, $98.21 \%, 63.16 \%$, and $89.33 \%$ for integrated IHC. In the cohort of follicular neoplasms (FN), they were $30.43 \%, 77.77 \%$, $77.77 \%, 30.43 \%$, and $43.75 \%$ for CK19; $73.91 \%, 66.67 \%, 85.00 \%, 50.00 \%$, and $71.88 \%$ for galectin-3; $26.09 \%, 88.89 \%$, $85.71 \%, 32.00 \%$, and $43.75 \%$ for HBME- $1 ; 26.09 \%, 100.00 \%, 100.00 \%, 34.62 \%$, and $46.88 \%$ for CD $56 ; 52.17 \%, 88.89 \%$, $92.31 \%, 42.11 \%$, and $62.50 \%$ for NGS; $82.61 \%, 66.67 \%, 86.36 \%, 60.00 \%$, and $78.13 \%$ for integrated IHC; and $100 \%$, $66.67 \%, 88.46 \%, 100 \%$, and $90.63 \%$ for integrated IHC-NGS. The application of biomarkers in distinguishing uncertain CNB samples of thyroid nodules is available and capable. CD56 negative or NGS positive suggests malignancy strongly for both FN and non-FN-lesion, which may be used as a "rule in" tool. The negative predictive value of the integrated IHC and the integrated IHC-NGS implies a high possibility to be benign for non-FN-lesion and FN separately, which can work as a "rule out" tool. Considering the balance of specificity and sensitivity, NGS is the best for non-FN-lesion and the integrated IHC-NGS is the best for FN.
\end{abstract}

Keywords Thyroid nodule $\cdot$ Core needle biopsy $\cdot$ Uncertain $\cdot$ Biomarker

Yan Xiong

yanxiong1109@163.com

1 Department of Pathology, Peking University First Hospital, 7 Xishiku Street, Xicheng District, Beijing 100034, China

2 Department of Pathology, Tangshan Gongren Hospital, 27 Wenhua Road, Lubei District, Tangshan 063000, Hebei, China

3 Department of Biostatistics, Peking University First Hospital, 7 Xishiku Street, Xicheng District, Beijing 100034, China

\section{Background}

Thyroid nodules are a common disease of the endocrine system. The prevalence is 20 to $76 \%$ in the Chinese population as identified by high-resolution ultrasound, and 5 to $15 \%$ of nodules are malignant [1]. It is crucial to screen these malignant cases for further treatment. The biopsy techniques involved in thyroid nodules' preoperative diagnosis include fine-needle biopsy (FNB) and core needle biopsy (CNB). FNB has been used worldwide for many years, and CNB has 
been used more and more frequently in Asia in the recent 10 years $[2,3]$. Several large single-center studies have shown no significant differences between FNB and CNB in terms of pain, tolerability, or complications due to the advances in CNB devices and the development of high-resolution ultrasound [4]. Compared to FNB, the morphology of cells and architectures of the tumors can be seen in the CNB samples, giving more support to pathologists to make a correct diagnosis. Published studies have shown that the accuracy of CNB for the thyroid nodules' preoperative diagnosis was higher than of FNB [5]. However, approximately 5-20\% of CNB samples are still uncertain of being benign or malignant based on morphology alone [5, 6]. Compared to FNB, samples collected by CNB are more accessible for various testing methods. Therefore, studying biomarkers' application in distinguishing uncertain CNB samples of thyroid nodules is a practical need. We retrieved 107 cases of thyroid nodules with uncertain CNB samples and matched resected specimens. Taking the matched resected specimens' diagnosis as the gold standard, we studied biomarkers' capability to distinguish uncertain CNB samples.

\section{Methods}

\section{Patients and samples}

Patients of thyroid nodules with both CNB and matched resected specimens treated at Peking University First Hospital between January 2015 and December 2020 were reviewed. CNB was used as the first-line preoperative diagnosis in all patients without prior FNB according to publication protocol [7]. The Peking University First Hospital Ethics Committee approved the usage of all patient samples and clinical data and an informed consent exemption (ethical approval no.: (2018) Research No. 147).

\section{Pathological review}

All hematoxylin and eosin (HE) staining slides were separately reviewed by two pathologists blinded to the original diagnoses. The CNB samples were diagnosed according to the Korean proposal: (I) nondiagnostic or unsatisfactory; (II) benign lesion; (III) indeterminate lesion; (IV) follicular neoplasm; (V) suspicious for malignancy; and (VI) malignant (Table 1) [8]. Cases classified as III-V were retrieved as "uncertain." The resected samples were diagnosed according to the 2017 WHO classification of tumors of endocrine organs (4th): conventional papillary thyroid carcinoma (CPTC), follicular variant papillary thyroid carcinoma (FVPTC), follicular thyroid carcinoma (FTC), follicular adenoma (FA), nodular hyperplasia $(\mathrm{NH})$, and thyroiditis [9]. The cases with inconsistent diagnoses were reviewed, and agreements were achieved by discussion. Furthermore, we divided the cohort into two groups, i.e., the follicular neoplasm (FN) and the non-follicular-neoplasm-lesion (nonFN-lesion), to see if the biomarkers' efficiency was different. The FN included FTC and FA. The non-FN-lesion included CPTC, FVPTC, NH, and thyroiditis.

\section{Immunohistochemistry stain}

The primary antibodies included antibodies against CK19 (Dako, Clone RCK108), galectin-3 (Invitrogen, A3A12), HBME-1 (Dako, Clone HBME-1), and CD56 (Dako, Clone 123C3). The antigen retrieval buffer was EDTA (pH 9.0), the temperature was $98{ }^{\circ} \mathrm{C}$, and the duration was $20 \mathrm{~min}$. We used EnVision FLEX + Mouse LINKER to amplify the signal, the EnVision FLEX Mini Kit to visualize the immunohistochemistry (IHC) reaction, and the Autostainer Link 48 (Agilent Technologies, Santa Clara, CA, USA) to complete the procedure. The normal thyroid follicles around the nodules were the best IHC staining and evaluation controls for CD56. For CK19, galectin-3, and HBME-1, the known positive samples were put side by side with the target samples on each slide as controls.

\section{Scoring the results of a single IHC biomarker}

Tumors with membranous \pm cytoplasmic reactivity for CK19 in more than $10 \%$ of cells with strong intensity were considered positive. Tumors with cytoplasmic + nuclear reactivity for galectin-3 and membranous reactivity for HBME-1 or CD56 in more than $10 \%$ of cells were deemed positive regardless of intensity [10].

\section{Integrating IHC markers}

The cohort positive of integrated IHC consisted of two groups: The first was CD56 negative no matter whether CK19, galectin-3, and HBME-1 were stained or not; The second was CD56 positive and the other markers simultaneously positive. The cutoff of simultaneously positive markers was different in the different panels. The first panel, named IHC-COMB 1, required all three simultaneously positive; the second panel, named IHC-COMB2, required at least two, and the third panel, named IHC-COMB3, required at least one.

\section{Next-generation sequencing}

The percentage of tumor components in the CNB samples was recorded. Genomic DNA was extracted from unstained 5- $\mu \mathrm{m}$-thick paraffin-embedded sections using the QIAamp DNA FFPE Tissue Kit (Qiagen, Hilden, Germany) following the manufacturer's instructions. 
Table 1 Diagnostic categories of thyroid core needle biopsy proposed by the Korean Thyroid Association [5]

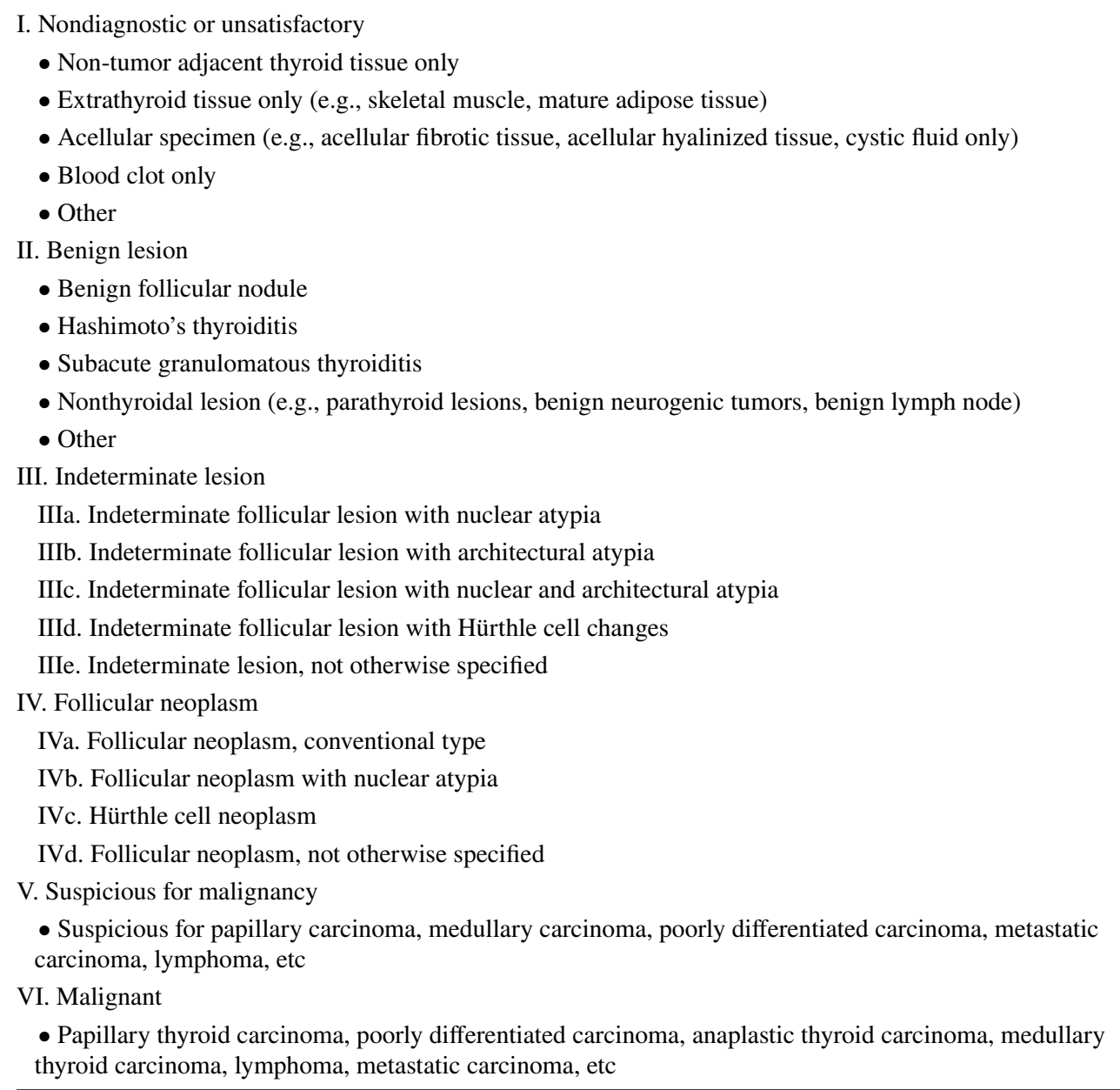

After extraction, DNA quality was evaluated by $1 \%$ agarose gel electrophoresis. The concentration of all samples was quantitated by a NanoDrop system (Invitrogen Life Technologies, Carlsbad, CA, USA) and Qubit Fluorometer (Invitrogen Life Technologies).

Targeted next-generation sequencing (NGS) was conducted using an OncoAim ${ }^{\circledR}$ thyroid cancer multigene assay kit (Singlera Genomics, Inc., Shanghai, China) that detected 26 genes (Table 2). According to the kit protocol, $50 \mathrm{ng}$ of DNA for each sample was used to generate sequencing libraries. DNA was fragmented by $5 \times$ WGS Fragmentation Mix (Qiagen, Beverly, MA, USA). After quality control and quantification, the library product was sequenced using $150 \mathrm{bp}$ paired-end runs on the NextSeq 500 platform (Illumina, Inc., San Diego, CA, USA). Sequencing data were then aligned to the reference human genome (hg19). Read mapping, quality control, variant calling, and genotyping were performed automatically using the tool kit supplied in the OncoAim ${ }^{\circledR}$ Kit (Singlera). The minimum confidence threshold for variant calling was set to $5 \%$. Variant functional annotation was performed with the ENSEMBL Variant Effect Predictor tool.
Based on ClinVar (Version 20,280,919), the result was marked as pathogenic, likely pathogenic, uncertain significance, likely benign, benign, or inconclusive. We recorded "confirmed pathogenic" or "likely pathogenic" as NGS positive.

\section{Integrating IHC and NGS}

The cohort positive of integrated IHC-NGS consisted of two groups: The first was NGS positive no matter whether the IHC markers were stained or not; The second was NGS negative and at least one of four IHC markers positive.

\section{Comparison between biomarkers' results of CNB samples and classification of matched resected specimens}

The results of biomarkers detected on CNB samples were compared to the classification of matched resected specimens. 
Table 2 Genes detected by OncoAim ${ }^{\circledR}$ thyroid cancer multigene assay kit

\begin{tabular}{|c|c|c|c|}
\hline \multirow[t]{2}{*}{ Gene } & \multirow[t]{2}{*}{ Transcript } & \multicolumn{2}{|l|}{ Variation type } \\
\hline & & Mutation & Fusion \\
\hline$B R A F$ & NM_004333 & Exon 15 & Introns $7-10$ \\
\hline$R E T$ & NM_020975 & Exons $7-16$ & Introns $10-11$ \\
\hline$N R A S$ & NM_002524 & Exons $2-3$ & - \\
\hline$K R A S$ & NM_033360 & Exons 2-4 & - \\
\hline$H R A S$ & NM_176795 & Exons 2-3 & - \\
\hline$A K T 1$ & NM_005163 & Exons $2-7$, exons 9-12 & - \\
\hline ATM & NM_000051 & All exons & - \\
\hline$C N N B 1$ & NM_001904 & All exons & - \\
\hline TSHR & NM_000369 & All exons & - \\
\hline$A P C$ & NM_000038 & All exons & - \\
\hline$T T N$ & NM_001256850 & All exons & - \\
\hline$T G$ & NM_003235 & All exons & - \\
\hline$R B 1$ & NM_000321 & All exons & - \\
\hline MEN1 & NM_000244 & All exons & - \\
\hline PDGFRA & NM_006206 & All exons & - \\
\hline$P I K 3 C A$ & NM_006218 & All exons & - \\
\hline$C D K N 2 A$ & NM_000077 & All exons & - \\
\hline EIF1AX & NM_001412 & All exons & - \\
\hline PTEN & NM_000314 & Exons 5-8 & - \\
\hline GNAS & NM_000516 & Exons 8-9 & - \\
\hline TP53 & NM_000546 & Exons 5-9 & - \\
\hline$T E R T$ & NM_198253 & $\begin{array}{l}\text { Promoter (chr5:1,295,183- } \\
\quad 1,295,302)\end{array}$ & - \\
\hline PPARG & NM_005037 & - & Intron 1 \\
\hline NTRK1 & NM_002529 & - & Intron 9, exon 12 \\
\hline NTRK3 & NM_002530 & - & Intron 13 \\
\hline$A L K$ & NM_004304 & - & Intron 16 , intron 19 \\
\hline
\end{tabular}

\section{Statistical analysis}

We put resected samples classified as CPTC, FVPTC, and FTC into a single group as "malignant" and thyroiditis, $\mathrm{NH}$, and FA into another group as "benign" for statistical analysis. Taking the resected specimens' classification as the gold standard, the sensitivity, specificity, positive predictive value (PPV), negative predictive value (NPV), and accuracy of each biomarker and various integrated panels for discriminating malignancy from benignity were calculated.

\section{Results}

\section{Patients}

The study included 107 patients. Of them, 27 were males and 80 were females, with ages ranging from 20 to 82 and a mean age of 50. Sixty-five patients were younger than 55, thirty-nine were older than 55, and three were 55.

\section{Histological classification}

The CNB samples included 40 (37.4\%) cases of indeterminate, 32 (29.9\%) cases of follicular neoplasm, and 35 (32.7\%) cases of suspicious malignancy.

Twenty-two (20.6\%) resected specimens were classified as benign, including 9 (8.4\%) cases of NH, 4 (3.7\%) cases of thyroiditis, and $9(8.4 \%)$ cases of FA. Eighty-five (79.4\%) resected specimens were classified as malignant, including 35 (32.7\%) cases of CPTC, 27 (25.2\%) cases of FVPTC, and $23(21.5 \%)$ cases of FTC.

Of the 40 cases classified as indeterminate on CNB samples, the matched resected samples were classified as thyroiditis for 4 cases, NH for 9 cases, and FVPTC for 27 cases. Of the 32 cases classified as follicular neoplasm on CNB samples, the matched resected samples were classified as FA for 9 cases and FTC for 23 cases. All 35 cases 
Table 3 Comparison between classification of CNB samples and classification of matched resected specimens based on morphology alone

\begin{tabular}{lllll}
\hline $\begin{array}{l}\text { Classification of } \\
\text { resected samples }\end{array}$ & \multicolumn{4}{l}{$\begin{array}{l}\text { Classification of CNB samples based on mor- } \\
\text { phology, no }\end{array}$} \\
\cline { 2 - 5 } & $\begin{array}{l}\text { Indetermi- } \\
\text { nate lesion }\end{array}$ & $\begin{array}{l}\text { Follicular } \\
\text { neoplasm }\end{array}$ & $\begin{array}{l}\text { Suspicious for } \\
\text { malignancy }\end{array}$ & Total \\
\hline NH & 9 & 0 & 0 & 9 \\
Thyroiditis & 4 & 0 & 0 & 4 \\
FA & 0 & 9 & 0 & 9 \\
CPTC & 0 & 0 & 35 & 35 \\
FVPTC & 27 & 0 & 0 & 27 \\
FTC & 0 & 23 & 0 & 23 \\
Total & 40 & 32 & 35 & 107 \\
\hline
\end{tabular}

$C N B$, core needle biopsy; $N H$, nodular hyperplasia; $F A$, follicular adenoma; $C P T C$, conventional papillary thyroid carcinoma; FVPTC, follicular variant of papillary thyroid carcinoma; FTC follicular thyroid carcinoma

classified as suspicious malignancy on CNB samples were classified as CPTC on resected samples (Table 3).

\section{Results of IHC}

Seventy-four cases $(69.16 \%)$ were positive for CK19, including 31 cases of indeterminate lesion, 9 cases of follicular neoplasm, and 34 cases of suspicious malignancy. Eighty-seven cases $(81.31 \%)$ were positive for galectin-3, including 33 cases of indeterminate lesion, 20 cases of follicular neoplasm, and 34 cases of suspicious malignancy. Fifty-eight cases (54.21\%) were positive for HBME-1, including 24 cases of indeterminate lesion, 7 cases of follicular neoplasm, and 27 cases of suspicious malignancy. Forty-seven cases $(43.93 \%)$ were negative for CD56, including 17 cases of indeterminate lesion, 6 cases of follicular neoplasm, and 24 cases of suspicious malignancy. Sixty-four cases $(59.81 \%)$ were positive for IHC-COMB1, including 23 cases of indeterminate lesion, 8 cases of follicular neoplasm, and 33 cases of suspicious malignancy. Seventy-six cases $(71.03 \%)$ were positive for IHC-COMB2, including 32 cases of indeterminate lesion, 11 cases of follicular neoplasm, and 33 cases of suspicious malignancy. Ninety-five cases $(88.79 \%)$ were positive for IHC-COMB3, including 38 cases of indeterminate lesion, 22 cases of follicular neoplasm, and 35 cases of suspicious malignancy (Table 4).

\section{Results of NGS}

Sixty-eight cases $(63.55 \%)$ were positive for NGS. The 41 cases with BRAF V600E mutation included 12 cases of indeterminate lesions and 29 cases of suspicious malignancy. The 8 cases with RAS mutation included 3 cases
Table 4 The results of immunohistochemistry of CNB samples

\begin{tabular}{|c|c|c|c|c|c|}
\hline \multicolumn{2}{|l|}{ IHC } & \multicolumn{4}{|c|}{ Classification of CNB samples based on morphology, no } \\
\hline Markers & Results & $\begin{array}{l}\text { Indeterminate } \\
\text { lesion }\end{array}$ & $\begin{array}{l}\text { Follicular neo- } \\
\text { plasm }\end{array}$ & $\begin{array}{l}\text { Suspicious for } \\
\text { malignancy }\end{array}$ & Total \\
\hline \multirow[t]{2}{*}{ CK19 } & Negative & 9 & 23 & 1 & 33 \\
\hline & Positive & 31 & 9 & 34 & 74 \\
\hline \multirow[t]{2}{*}{ Galectin-3 } & Negative & 7 & 12 & 1 & 20 \\
\hline & Positive & 33 & 20 & 34 & 87 \\
\hline \multirow[t]{2}{*}{ HBME-1 } & Negative & 16 & 25 & 8 & 49 \\
\hline & Positive & 24 & 7 & 27 & 58 \\
\hline \multirow[t]{2}{*}{ CD56 } & Positive & 23 & 26 & 11 & 60 \\
\hline & Negative & 17 & 6 & 24 & 47 \\
\hline \multirow[t]{2}{*}{ IHC-COMB 1} & Negative & 17 & 24 & 2 & 43 \\
\hline & Positive & 23 & 8 & 33 & 64 \\
\hline \multirow[t]{2}{*}{ IHC-COMB2 } & Negative & 8 & 21 & 2 & 31 \\
\hline & Positive & 32 & 11 & 33 & 76 \\
\hline \multirow[t]{2}{*}{ IHC-COMB3 } & Negative & 2 & 10 & 0 & 12 \\
\hline & Positive & 38 & 22 & 35 & 95 \\
\hline Total & & 40 & 32 & 35 & 107 \\
\hline
\end{tabular}

$C N B$, core needle biopsy; IHC, immunohistochemistry; IHC-COMB1, CD56 negative no matter whether CK19, galectin-3, and HBME-1 are positive or not/CD56 positive and all of CK19, galectin-3, and HBME-1 simultaneously positive; IHC-COMB2, CD56 negative no matter whether CK19, galectin-3, and HBME-1 are positive or not/CD56 positive and at least two of CK19, galectin-3, and HBME-1 simultaneously positive; IHC-COMB3, CD56 negative no matter whether CK19, galectin-3, and HBME-1 are positive or not/CD56 positive and at least one of CK19, galectin-3, and HBME-1 simultaneously positive 
Table 5 The results of OncoAim®-NGS of CNB samples

\begin{tabular}{|c|c|c|c|c|}
\hline \multirow[t]{2}{*}{ NGS } & \multicolumn{4}{|c|}{$\begin{array}{l}\text { Classification of CNB samples based on morphol- } \\
\text { ogy, no }\end{array}$} \\
\hline & $\begin{array}{l}\text { Indetermi- } \\
\text { nate lesion }\end{array}$ & $\begin{array}{l}\text { Follicular } \\
\text { neoplasm }\end{array}$ & $\begin{array}{l}\text { Suspicious for } \\
\text { malignancy }\end{array}$ & Total \\
\hline Negative & 15 & 19 & 5 & 39 \\
\hline BRAF V600E & 12 & 0 & 29 & 41 \\
\hline PTEN mutation & 0 & 1 & 0 & 1 \\
\hline ALK fusion & 1 & 0 & 0 & 1 \\
\hline RET fusion & 5 & 1 & 1 & 7 \\
\hline NTRK fusion & 4 & 0 & 0 & 4 \\
\hline RAS mutation & 3 & 5 & 0 & 8 \\
\hline PPAR $\gamma$ fusion & 0 & 1 & 0 & 1 \\
\hline TERT mutation & 0 & 4 & 0 & 4 \\
\hline $\begin{array}{l}\text { Non-V600E } \\
\text { BRAF mutation }\end{array}$ & 0 & 1 & 0 & 1 \\
\hline Total & 40 & 32 & 35 & 107 \\
\hline
\end{tabular}

$C N B$, core needle biopsy; $N G S$, next-generation sequencing of indeterminate lesion and 5 cases of follicular neoplasm. The 7 cases with RET fusion included 5 cases of indeterminate lesion, 1 case of follicular neoplasm, and 1 case of suspicious malignancy. All of the 4 cases with NTRK fusion were indeterminate lesions. The one case with ALK fusion was indeterminate lesion. All of the 4 cases with TERT mutation were follicular neoplasm. The rest of the three follicular neoplasms had PTEN mutation, PPAR $\gamma$ fusion, and non-V600E BRAF mutation separately (Table 5).

\section{Results of integrated IHC-NGS}

Ninety-nine cases $(92.52 \%)$ were positive for the integrated IHC-NGS, including 38 cases of indeterminate lesion, 26 cases of follicular neoplasm, and 35 cases of suspicious malignancy.
Table 6 Predictive value of biomarkers for all cases

\begin{tabular}{|c|c|c|c|c|c|c|c|c|c|}
\hline & \multirow[t]{2}{*}{ CNB samples, no } & \multicolumn{2}{|c|}{$\begin{array}{l}\text { Matched resected speci- } \\
\text { mens, no }\end{array}$} & \multirow[b]{2}{*}{ Total } & \multicolumn{5}{|c|}{ Predictive value, $\%$} \\
\hline & & Benignity & Malignancy & & Sen & Spe & PPV & NPV & $\mathrm{AC}$ \\
\hline \multirow[t]{2}{*}{ CK19 } & Negative & 14 & 19 & 33 & 77.65 & 63.63 & 89.19 & 42.42 & 74.77 \\
\hline & Positive & 8 & 66 & 74 & & & & & \\
\hline \multirow[t]{2}{*}{ Galectin-3 } & Negative & 11 & 9 & 20 & 89.41 & 50.00 & 87.36 & 55.00 & 81.31 \\
\hline & Positive & 11 & 76 & 87 & & & & & \\
\hline \multirow[t]{2}{*}{ HBME-1 } & Negative & 18 & 31 & 49 & 63.53 & 81.81 & 93.10 & 36.73 & 67.29 \\
\hline & Positive & 4 & 54 & 58 & & & & & \\
\hline \multirow[t]{2}{*}{ CD56 } & Positive & 22 & 38 & 60 & 55.29 & 100 & 100 & 36.67 & 64.49 \\
\hline & Negative & 0 & 47 & 47 & & & & & \\
\hline \multirow[t]{2}{*}{ IHC-COMB1 } & Negative & 20 & 23 & 43 & 72.94 & 90.91 & 96.88 & 46.51 & 76.64 \\
\hline & Positive & 2 & 62 & 64 & & & & & \\
\hline \multirow[t]{2}{*}{ IHC-COMB2 } & Negative & 15 & 16 & 31 & 81.18 & 68.18 & 90.79 & 48.39 & 78.50 \\
\hline & Positive & 7 & 69 & 76 & & & & & \\
\hline \multirow[t]{2}{*}{ IHC-COMB3 } & Negative & 8 & 4 & 12 & 95.29 & 36.36 & 85.26 & 66.67 & 83.18 \\
\hline & Positive & 14 & 81 & 95 & & & & & \\
\hline \multirow[t]{2}{*}{ NGS } & Negative & 20 & 17 & 37 & 80.00 & 90.90 & 97.14 & 54.05 & 82.24 \\
\hline & Positive & 2 & 68 & 70 & & & & & \\
\hline \multirow[t]{3}{*}{ IHC-NGS } & Negative & 8 & 0 & 8 & 100 & 36.36 & 85.86 & 100 & 86.92 \\
\hline & Positive & 14 & 85 & 99 & & & & & \\
\hline & Total & 22 & 85 & 107 & & & & & \\
\hline
\end{tabular}

$C N B$, core needle biopsy; Sen, sensitivity; Spe, specificity; $P P V$, positive predictive value; $N P V$, negative predictive value; $A C$, accuracy; IHC-COMB1, CD56 negative no matter whether CK19, galectin-3, and HBME-1 are positive or not/CD56 positive and all of CK19, galectin-3, and HBME-1 simultaneously positive; IHC-COMB2, CD56 negative no matter whether CK19, galectin-3, and HBME-1 are positive or not/ CD56 positive and at least two of CK19, galectin-3, and HBME-1 simultaneously positive; IHC-COMB3, CD56 negative no matter whether CK19, galectin-3, and HBME-1 are positive or not/CD56 positive and at least one of CK19, galectin-3, and HBME-1 simultaneously positive; IHC-NGS, immunohistochemistry and next-generation sequencing combination 

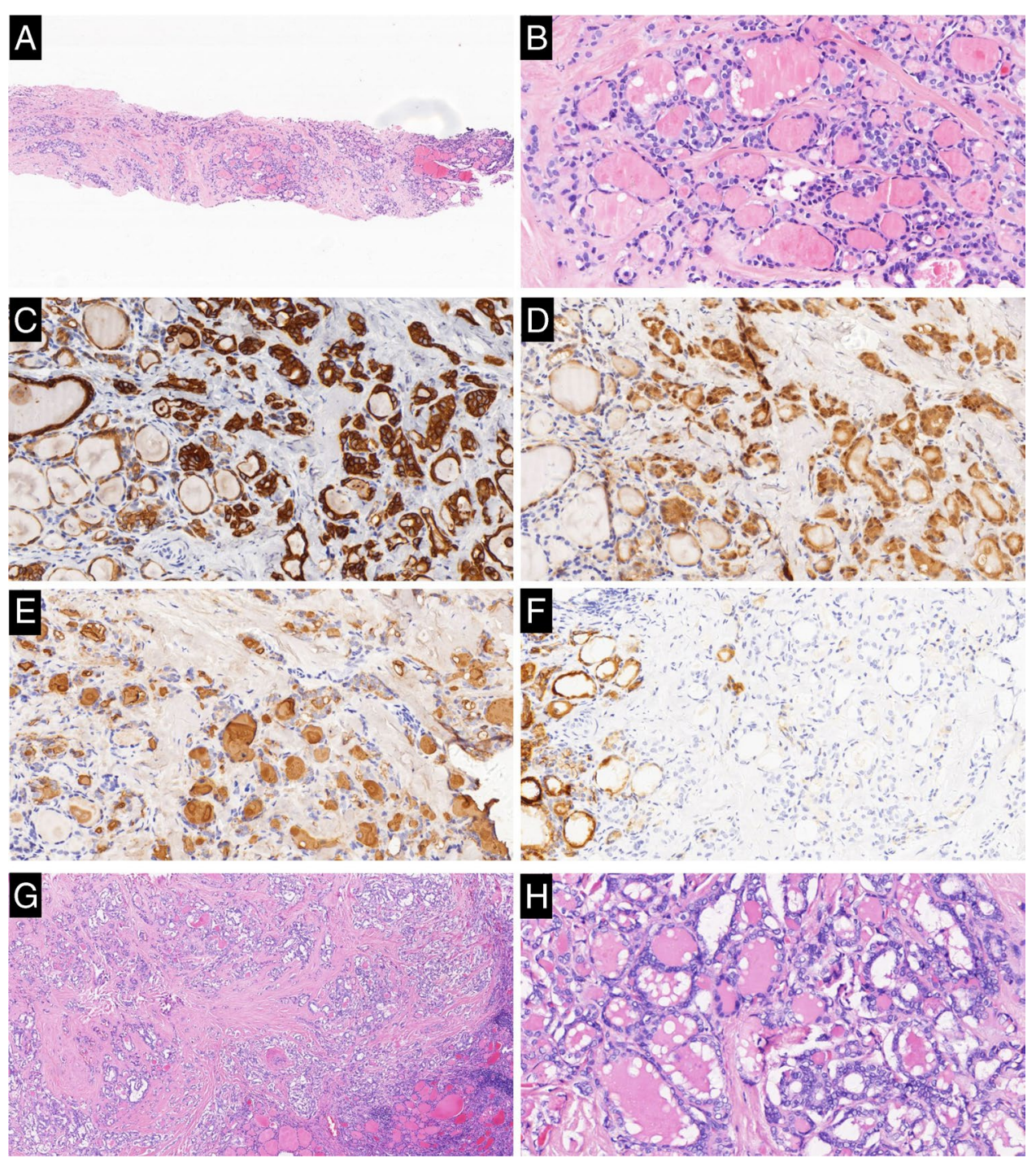

Fig. 1 Case classified as indeterminate lesion in the CNB sample while follicular variant of papillary thyroid carcinoma in the matched resected specimen. a Tumor in the CNB sample is entirely composed of follicular structures $(\mathrm{H} \& \mathrm{E} \times 40)$. b High magnification of the lesion shows the follicular structures lined by cells with nuclei scored $1(H \& E \times 200)$. c Cytoplasm and membrane of tumor cells in the CNB sample are diffusely reactive for CK19 with strong intensity, while the normal follicular cells are reactive with weak intensity $(\mathrm{CK} 19 \times 200)$. d Cytoplasm and nuclei of tumor cells in the CNB sample are diffusely reactive for galectin-3 with strong intensity, while the normal follicular cells are nonreactive (galectin-3×200). e Membranes of tumor cells in the CNB sample are partially (about $30 \%$ ) reactive for HBME-1 with intermediate intensity, while the normal follicular cells are nonreactive $(\mathrm{HBME}-1 \times 200)$. f Tumor cells in the CNB samples are nonreactive for CD56, while membrane and cytoplasm of the normal follicular cells are diffusely reactive with strong intensity $(\mathrm{CD} 56 \times 200)$. $\mathbf{g}$ Tumor in the matched resected specimen is entirely composed of follicular structures. (H\&E $\times 40)$. $\mathbf{h}$ High magnification of the lesion shows the follicular structures lined by cells with nuclei scored $3(\mathrm{H} \& \mathrm{E} \times 200)$

\section{Comparison between biomarkers' results of CNB samples and classification of matched resected specimens}

Of the 74 cases positive of CK19 on CNB samples, 66 were classified as malignant and 8 were classified as benign on the matched resected samples. Of the 87 cases positive of galectin-3 on CNB samples, 76 were classified as malignant and 11 were classified as benign on the matched resected samples. Of the 58 cases positive of HBME- 1 on CNB samples, 54 were classified as malignant and 4 were classified as benign on the matched resected samples. All 47 cases of CD56 negative on CNB samples were diagnosed as malignant on the matched resected sample too (Table 6) (Figs. 1, 2, and 3). 
Fig. 2 Case classified as indeterminate lesion in the CNB sample while conventional papillary thyroid carcinoma with a follicular predominant growth pattern in the matched resected specimen. a Tumor in the CNB sample is entirely composed of follicular structures $(\mathrm{H} \& \mathrm{E} \times 40)$. b High magnification of the lesion shows the follicular structures lined by cells with nuclei scored $2(\mathrm{H} \& \mathrm{E} \times 200)$. c Cytoplasm and membrane of tumor cells in the CNB sample are diffusely reactive for CK19 with strong intensity, while the normal follicular cells are nonreactive $(\mathrm{CK} 19 \times 200)$. d Cytoplasm and nuclei of tumor cells in the CNB sample are diffusely reactive for galectin-3 with strong intensity, while the normal follicular cells are nonreactive (galectin-3×200). e Membranes of tumor cells in the CNB sample are diffusely reactive for HBME-1 with strong intensity, while the normal follicular cells are nonreactive (HBME-1 $\times 200)$. f Tumor cells in the CNB samples are nonreactive for CD56, while membrane and cytoplasm of the normal follicular cells are diffusely reactive with intermediate intensity $($ CD56×200) $\mathbf{g}, \mathbf{h}$, Tumor in the matched resected specimen is almost entirely composed of follicular structures, except of focal papillary structure $(\mathrm{H} \& \mathrm{E} \times 40)$. i High magnification of the lesion shows the follicular structures lined by cells with nuclei scored 3 (H\&E× 200). $\mathbf{j}$ High magnification of the lesion shows the papillary structures lined by cells with nuclei scored $3(\mathrm{H} \& \mathrm{E} \times 200)$
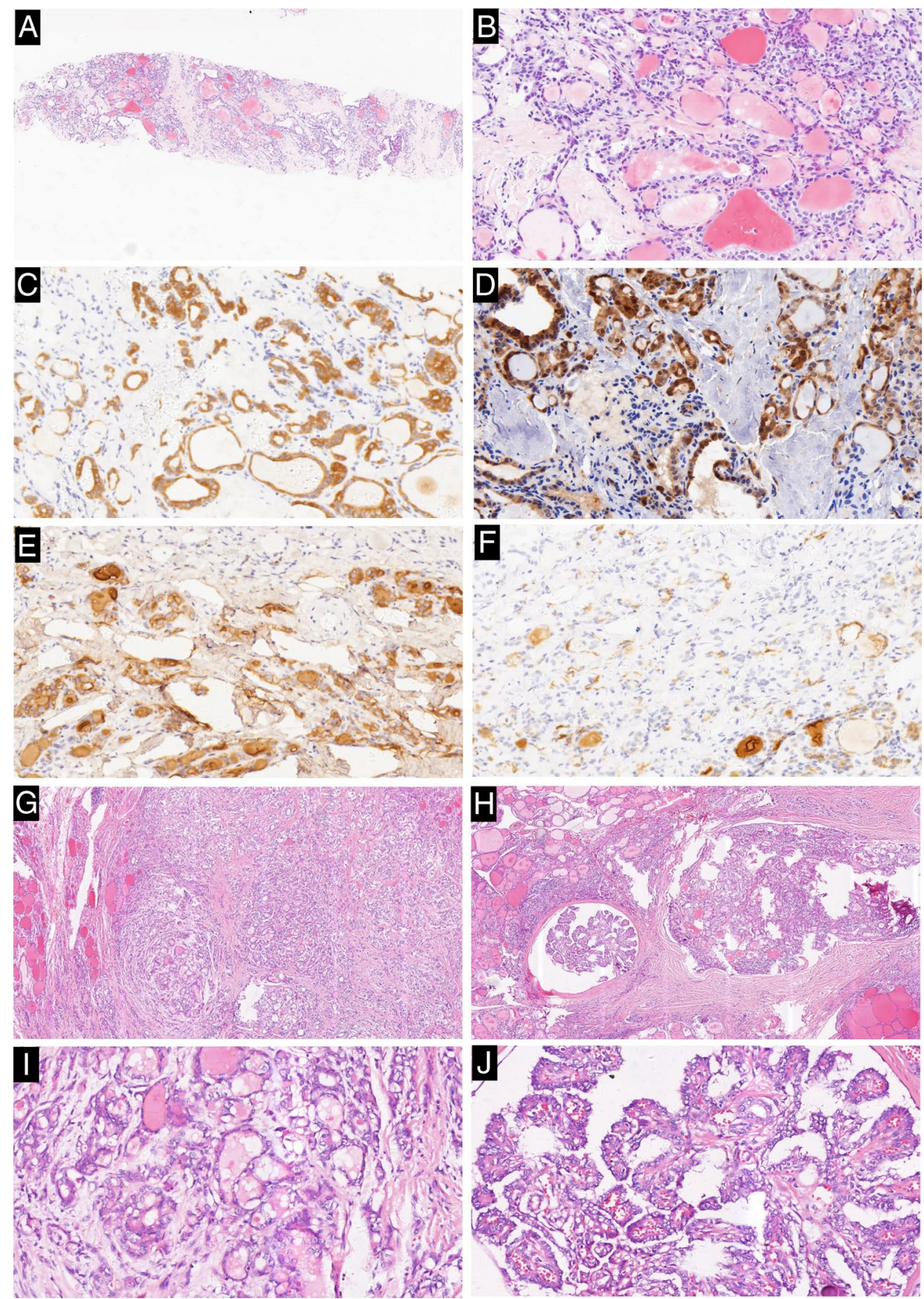

Of the 64 cases of IHC-COMB1 positive on CNB samples, 62 were classified as malignant and two were classified as benign on the matched resected samples. Of the 76 cases of IHC-COMB2 positive on CNB samples, 69 were classified as malignant and seven were classified as benign on the matched resected samples. Of the 95 cases of IHC-COMB3 positive on $\mathrm{CNB}$ samples, 81 were classified as malignant and 14 were classified as benign on the matched resected specimens (Table 6).
Of the 70 cases of NGS positive on CNB samples, 68 were classified as malignant and two were classified as benign on the matched resected specimens (Table 6). The 41 cases with BRAF V600E mutation included 29 cases of CPTC and 12 cases of FVPTC. The 8 cases with RAS mutation included 5 cases of FTC, 2 cases of FVPTC, and 1 case of NH. The 7 cases with RET fusion included 5 cases of FVPTC, 1 case of CPTC, and 1 case of FTC. All of the 4 cases with NTRK fusion were FVPTC. All of the 4 cases with TERT mutation were FTC. The one case with 
Fig. 3 Case classified as follicular neoplasm in the CNB sample while follicular thyroid carcinoma in the matched resected specimen. a The CNB specimen shows a microfollicular proliferative lesion with a thick fibrous capsule separating it from the normal follicles $(\mathrm{H} \& \mathrm{E} \times 40)$. b High magnification of the lesion shows the follicular structures lined by cells with nuclei scored 0 (H\&E $\times 200)$. c Tumor cells in the CNB sample are negative for CK19 $($ CK19 $\times 200)$. d Tumor cells in the CNB sample are negative for galectin-3 (galectin-3×200). e Tumor cells in the CNB sample are negative for HBME-1 (HBME-1 × 200). f Tumor cells in the CNB samples are positive for CD56 $(\mathrm{CD} 56 \times 200)$. $\mathrm{g}$ Tumor in the matched resected specimen is encapsulated with capsular infiltration and entirely composed of follicular structures $(\mathrm{H} \& \mathrm{E} \times 40)$. h High magnification of the lesion shows the follicular structures lined by cells with nuclei scored $0(\mathrm{H} \& \mathrm{E} \times 200)$
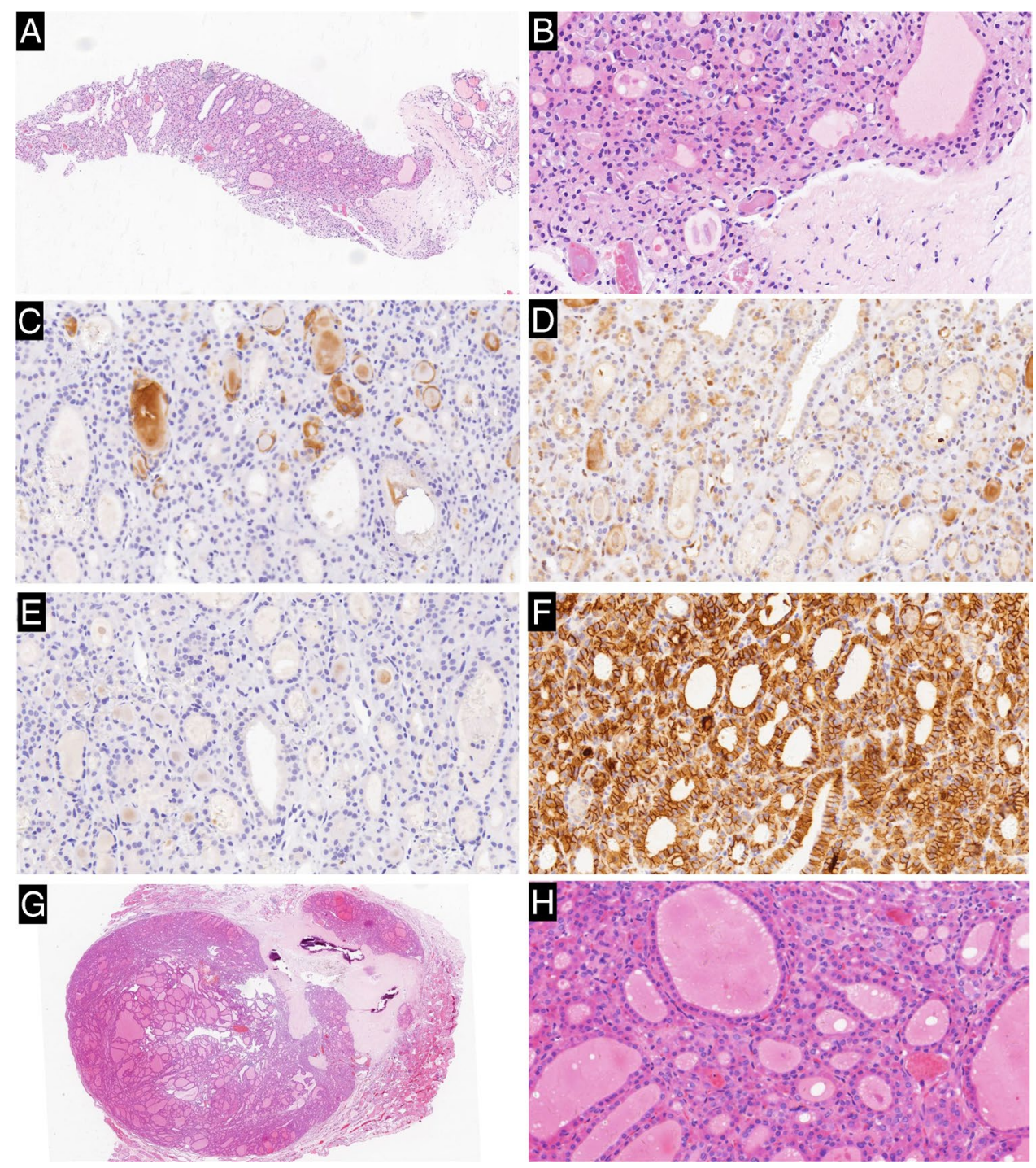

ALK fusion was FVPTC. The one case with PTEN mutation was FTC. The one case with PPAR $\gamma$ fusion was FTC. The one case with non-V600E BRAF mutation was FA.

Of the 99 cases positive of integrated IHC-NGS on CNB samples, 85 were classified as malignant and 14 were classified as benign on the matched resected samples (Table 6), including 35 cases of CPTC, 27 cases of FVPTC, 23 cases of FTC, 8 cases of NH, 3 cases of FA, and 3 cases of thyroiditis.

\section{Predictive value of biomarkers}

Taking the classification of the matched resected specimens as the gold standard, the sensitivity, specificity, PPV, NPV, and accuracy for preoperative malignancy evaluation for the whole cohort were $77.65 \%, 63.64 \%, 89.19 \%, 42.43 \%$, and $74.77 \%$ for CK19; 89.42\%, 50.00\%, 87.36\%, 55.00\%, and $81.31 \%$ for galectin- $3 ; 63.53 \%, 81.82 \%, 93.10 \%, 36.73 \%$, and $67.29 \%$ for HBME- $1 ; 55.29 \%, 100.00 \%, 100.00 \%, 36.67 \%$, and $64.49 \%$ for CD56; $80.00 \%, 90.91 \%, 97.14 \%, 54.05 \%$, and $82.24 \%$ for NGS; $72.94 \%, 90.91 \%, 96.88 \%, 46.51 \%$, and $76.64 \%$ for IHCCOMB1; $81.18 \%, 68.18 \%, 90.79 \%, 48.39 \%$, and $78.50 \%$ for IHC-COMB2; 95.29\%, 36.36\%, 85.26\%, 66.67\%, and 83.18\% for IHC-COMB3; and $100.00 \%, 36.36 \%, 85.86 \%, 100.00 \%$, and $86.92 \%$ for integrated IHC-NGS (Table 6).

The sensitivity, specificity, PPV, NPV, and accuracy for preoperative malignancy evaluation of non-FN-lesions were $95.16 \%, 53.85 \%, 90.77 \%, 70.00 \%$, and $88.00 \%$ for CK19; $95.16 \%, 38.46 \%, 88.06 \%, 62.50 \%$, and $85.33 \%$ for galectin-3; $77.42 \%, 76.92 \%, 94.12 \%, 41.67 \%$, and $58.00 \%$ for HBME-1; 66.13\%, $100.00 \%, 100.00 \%, 38.24 \%$, and $72.00 \%$ for CD56; $90.32 \%, 92.31 \%, 98.25 \%, 66.67 \%$, and $90.67 \%$ for NGS; $88.71 \%, 92.30 \%, 98.21 \%, 63.16 \%$, and $89.33 \%$ for IHC-COMB1; $96.77 \%, 61.54 \%, 92.31 \%, 80.00 \%$, and $90.67 \%$ for IHC-COMB2; $100.00 \%, 15.38 \%, 84.93 \%$, $100.00 \%$, and $85.33 \%$ for IHC-COMB3; and $100.00 \%$, $36.36 \%, 85.86 \%, 100.00 \%$, and $86.92 \%$ for integrated IHCNGS (Table 7). 
Table 7 Predictive value of biomarkers for cases of nonfollicular-neoplasm-lesion

\begin{tabular}{|c|c|c|c|c|c|c|c|c|c|}
\hline & \multirow[t]{2}{*}{ CNB samples, no } & \multicolumn{2}{|c|}{$\begin{array}{l}\text { Matched resected sam- } \\
\text { ples, no }\end{array}$} & \multirow[b]{2}{*}{ Total } & \multicolumn{5}{|c|}{ Predictive value, $\%$} \\
\hline & & Benignity & Malignancy & & Sen & Spe & PPV & NPV & $\mathrm{AC}$ \\
\hline \multirow[t]{2}{*}{ CK19 } & Negative & 7 & 3 & 10 & 95.16 & 53.85 & 90.77 & 70.00 & 88.00 \\
\hline & Positive & 6 & 59 & 65 & & & & & \\
\hline \multirow[t]{2}{*}{ Galectin-3 } & Negative & 5 & 3 & 8 & 95.16 & 38.46 & 88.06 & 62.50 & 85.33 \\
\hline & Positive & 8 & 59 & 67 & & & & & \\
\hline \multirow[t]{2}{*}{ HBME-1 } & Negative & 10 & 14 & 24 & 77.42 & 76.92 & 94.12 & 41.67 & 58.00 \\
\hline & Positive & 3 & 48 & 51 & & & & & \\
\hline \multirow[t]{2}{*}{ CD56 } & Positive & 13 & 21 & 34 & 66.13 & 100 & 100 & 38.24 & 72.00 \\
\hline & Negative & 0 & 41 & 41 & & & & & \\
\hline \multirow[t]{2}{*}{ IHC-COMB1 } & Negative & 12 & 7 & 19 & 88.71 & 92.30 & 98.21 & 63.16 & 89.33 \\
\hline & Positive & 1 & 55 & 56 & & & & & \\
\hline \multirow[t]{2}{*}{ IHC-COMB2 } & Negative & 8 & 2 & 10 & 96.77 & 61.54 & 92.31 & 80.00 & 90.67 \\
\hline & Positive & 5 & 60 & 65 & & & & & \\
\hline \multirow[t]{2}{*}{ IHC-COMB3 } & Negative & 2 & 0 & 2 & 100 & 15.38 & 84.93 & 100 & 85.33 \\
\hline & Positive & 11 & 62 & 73 & & & & & \\
\hline \multirow[t]{2}{*}{ NGS } & Negative & 12 & 6 & 18 & 90.32 & 92.31 & 98.25 & 66.67 & 90.67 \\
\hline & Positive & 1 & 56 & 57 & & & & & \\
\hline \multirow[t]{3}{*}{ IHC-NGS } & Negative & 2 & 0 & 2 & 100 & 15.38 & 84.93 & 100 & 85.33 \\
\hline & Positive & 11 & 62 & 73 & & & & & \\
\hline & Total & 13 & 62 & 75 & & & & & \\
\hline
\end{tabular}

$C N B$, core needle biopsy; Sen, sensitivity; Spe, specificity; $P P V$, positive predictive value; $N P V$, negative predictive value; $A C$, accuracy; IHC-COMB1, CD56 negative no matter whether CK19, galectin-3, and HBME-1 are positive or not/CD56 positive and all of CK19, galectin-3, and HBME-1 simultaneously positive; IHC-COMB2, CD56 negative no matter whether CK19, galectin-3, and HBME-1 are positive or not/ CD56 positive and at least two of CK19, galectin-3, and HBME-1 simultaneously positive; IHC-COMB3, CD56 negative no matter whether CK19, galectin-3, and HBME-1 are positive or not/CD56 positive and at least one of CK19, galectin-3, and HBME-1 simultaneously positive; IHC-NGS, immunohistochemistry and next-generation sequencing combination
The sensitivity, specificity, PPV, NPV, and accuracy for preoperative malignancy evaluation of $\mathrm{FN}$ were $30.43 \%$, $77.77 \%, 77.77 \%, 30.43 \%$, and $43.75 \%$ for CK19; $73.91 \%$, $66.67 \%, 85.00 \%, 50.00 \%$, and $71.88 \%$ for galectin-3; $26.09 \%, 88.89 \%, 85.71 \%, 32.00 \%$, and $43.75 \%$ for HBME$1 ; 26.09 \%, 100.00 \%, 100.00 \%, 34.62 \%$, and $46.88 \%$ for CD56; $52.17 \%, 88.89 \%, 92.31 \%, 42.11 \%$, and $62.50 \%$ for NGS; $30.43 \%, 88.89 \%, 87.50 \%, 33.33 \%$, and $46.88 \%$ for IHC-COMB $1 ; 39.13 \%, 77.78 \%, 81.82 \%, 33.33 \%$, and $50.00 \%$ for IHC-COMB2; 82.61\%, 66.67\%, 86.36\%, $60.00 \%$, and $78.13 \%$ for IHC-COMB3; and $100.00 \%, 66.67 \%$, $88.46 \%, 100.00 \%$, and $90.63 \%$ for integrated IHC-NGS integrated(Table 8 ).

\section{Discussion}

Morphological changes, including nuclear score, architecture (papillary or follicular), and growth pattern (infiltrative or encapsulated), are critical for diagnosing thyroid tumors. Based on the criteria above, major cases can be diagnosed undoubtedly. However, some cases are difficult to determine based on histological morphology alone. Compared to resected specimens, the diagnoses of biopsies are more challenging. The uncertain diagnosis rate is $10-40 \%$ for FNB and 5-20\% for CNB [5]. Our comparative study between $\mathrm{CNB}$ and resected specimens of thyroid nodules showed that 74 of 578 cases could not be ascertained as malignant or benign based on the CNB sample's morphology alone [6]. The reason is that only follicles visible on CNB with atypical nuclei without normal tissue as a background make it impossible to differentiate FTC, FVPTC, and CPTC with a follicular predominant growth pattern from $\mathrm{FA}, \mathrm{NH}$, and thyroiditis. Therefore, studying the application of biomarkers in distinguishing uncertain biopsy samples is necessary.

Immunohistochemistry is the most popular ancillary technique used in pathological practice. Studies on resected specimens showed that CK19, galectin-3, HBME-1, and CD56 were very helpful in discriminating malignancy from benignity [10-13]. In Dunderovic et al.'s study, the sensitivity of CK19, galectin-3, HBME1 , and CD56 was $75.41 \%, 88.52 \%, 71.31 \%$, and $58.20 \%$, 


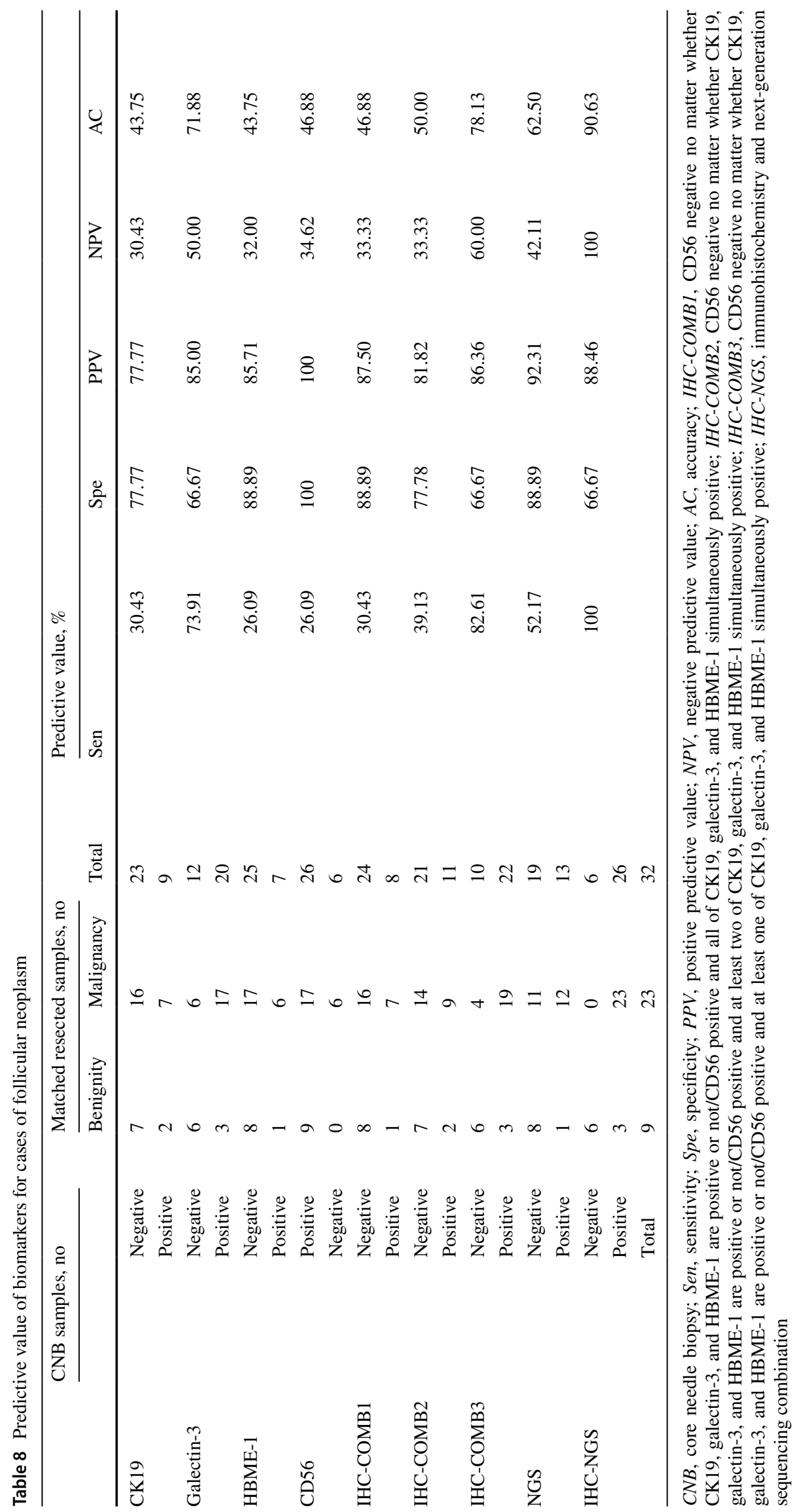


respectively, and the specificity of CK19, galectin-3, HBME-1, and CD56 was $70.89 \%, 64.56 \%, 84.81 \%$, and $92.41 \%$, separately [10]. Based on the knowledge above, it was supposed that IHC might play a role in improving the accuracy of diagnosing uncertain biopsy samples. We searched papers published in English in PubMed and found only one focusing on this topic. In this paper, Song et al. reported that the continued uncertain rate was $42.9 \%$ for FNB and $11.3 \%$ for CNB after IHC was applied [14].

Our study showed that, taking the resected specimens' diagnosis as the gold standard, biomarker's efficiency in determining the uncertain CNB samples as malignant or benign was various. Besides, even the same marker had a different power between FN and non-FN-lesions. The specificity of CD56 is perfect (100\%) for both FN and non-FN-lesions, but the sensitivity is low $(66.13 \%$ for nonFN-lesions and $26.09 \%$ for FN). Therefore, CD56 negative is particular for "ruling in" the malignant CNB samples; however, CD56 positive should not be used as the indicator of benignity. On the contrary, galectin-3 showed high sensitivity $(95.16 \%)$ for non-FN-lesions and moderate sensitivity $(73.91 \%)$ for FN but low specificity $(38.46 \%$ for non-FN-lesions and $66.67 \%$ for FN). Hence, galectin-3 negative could be highly suggestive of benignity for nonFN-lesions and cautiously used to support benignity for FN. Galectin-3 positive should not be used as the indicator of malignancy.

Given the limitation of a single marker, it is judicious to diagnose based on the integrated results. Considering that CD56 has perfect specificity but low sensitivity, the combination should precisely pick back the cases left out by CD56. Our study showed that keeping the CD56-negative cases in the cohort of malignancy and picking back the cases with CD56 positive and all of the other three markers simultaneously positive was a suitable strategy to balance the specificity (92.30\%) and sensitivity (88.71\%) for the nonFN-lesion. But for FN, none of the combined panels had apparent advantages over a single marker.

In the past 10 years, we have witnessed significant progress in the molecular pathology of thyroid carcinoma. In 2014, The Cancer Genome Atlas (TCGA) reported the comprehensive genomic characteristics of PTC. Ninetyseven percent of PTCs have unique molecular alterations, in which BRAF V600E mutations, RAS mutations, RET fusions, and TERT mutations are frequently detected, but EIF1AX mutations, ALK fusions, and NTRK1 or NTRK3 fusions are infrequent [15]. Subsequently, the genotypes of FTC, poorly differentiated thyroid carcinoma (PDTC), and anaplastic thyroid carcinoma (ATC) have also been reported. In FTC, RAS mutations, PPAR $\gamma$ fusions, and TERT mutations are frequently detected, but BRAF K601E mutations and EIF1AX mutations are infrequent. In PDTC and ATC, BRAF V600E mutations, RAS mutations, TERT mutations, and TP53 mutations are frequently detected [16-18]. Based on their understanding of thyroid carcinoma's mutational profile, researchers have tried to use diverse molecular approaches to improve diagnosing uncertain biopsy samples and have presented various published results. The sensitivity and specificity of gene testing for discriminating malignancy from benignity were $63-94 \%$ and 52-99\%, respectively, with FNB [19-21]. Regardless of how sensitive or specific it is, applying gene testing to FNB is inconvenient in clinical practice because specialized sample collection is required at the initial procedure. Besides, the morphology of FNB samples used in the molecular test is unknown. In contrast, CNB samples are routinely stored as paraffin-embedded blocks in which DNA can readily be extracted and morphology can be reviewed at any moment. In this case, gene testing is supposed to distinguish uncertain samples more practically and effectively on CNB than FNB.

Compared to FNB, the number of publications about $\mathrm{CNB}$ is minimal, and only a few single mutations have been reported [22-25]. In our research, uncertain CNB samples were detected by NGS using the commercial panel OncoAim ${ }^{\circledR}$, which detected 26 genes covering the major molecular alterations of thyroid carcinoma. The sample was recorded as NGS positive when confirmed pathogenic or likely pathogenic mutations were detected. Taking the diagnosis of the resected specimens as the gold standard, NGS is highly specific (92.31\%) and sensitive (90.32\%) for the nonFN-lesion, and highly specific (88.89\%) but low sensitive $(52.17 \%)$ for the FN. In other words, NGS's positive result suggests malignancy strongly for both non-FN and FN. But the negative result should be cautiously used as an indicator of benignity for non-FN-lesion and not be used as an indicator of benignity for FN. Taking PPV and NPV considered together, NGS's efficiency was high for non-FN-lesion and moderate for FN.

Because NGS is not a universal technique and different laboratories may use diverse gene panels, platforms, and methods, the working power of NGS depends largely on each laboratory's technical details. In practice, it is a suitable way for pathologists to interpret NGS results based on the knowledge integrating the literature's reports and own lab's data. All of the data and analysis about NGS in our research are based on the specific commercial tool OncoAim®.

In our study, there were 37 cases with NGS negative results on CNB samples. The diagnosis of their matched resected specimens was benign for 20 cases and malignant for 17 cases. The 17 malignant cases included 11 FTC cases, 5 CPTC cases, and 1 FVPTC case. Then, we detected the 17 cases' genes on matched resected specimens and found that 11 FTC cases were really negative and the other six were false negative, including 5 CPTC cases with BRAF V600E and one FVPTC with NRAS mutation. Furthermore, the CNB slides of the six 
false-negative cases were reviewed, and it is shown that very few tumor components (less than 5\%) are in them. In conclusion, the inherent features of gene mutations of thyroid tumors, especially follicular neoplasm, are considered the main reason for NGS's relatively low efficiency as a benign marker. The false-negative results due to the limitation of tumor quantity in CNB samples are another factor in weakening NGS's power to pick up benign cases, even though the influence is lower than FNB.

Fortunately, all six NGS false-negative cases were positive for CK19, galectin-3, and HBME-1 and negative for CD56 on CNB, which gave us the confidence to make a malignant diagnosis. So, IHC plays an essential role in these cases with NGS's false-negative results due to the limitation of tumor quantity in CNB samples.

For non-FN-lesions, either IHC or NGS can work well individually. Therefore, combining them is unnecessary and not cost-effective. On the contrary, neither of them is powerful enough for FN when used separately. So, designing an integrated panel for improving the predictive value is a practical need. Considering the treatment of FN recommended by the NCCN guideline [26], patients may benefit more from the safe "rule out" strategy than the precise "rule in" strategy. Based on this principle, we designed the integrated panel to keep the NGS positive cases in the cohort of malignancy and pick back the cases with at least one of four IHC markers positive. This panel can raise sensitivity and NPV to $100 \%$ and keep acceptable specificity (66.67\%) and PPV $(88.46 \%)$, which may be superior to use IHC or NGS separately. The negative FN cases are highly possible to be benign, and nodule surveillance may be recommended with a bit of worry.

Finally, although it is acknowledged that presenting the results as a risk of malignancy (ROM) than a binary fashion is more clinically valuable, such modification of ROM is currently unavailable due to limited number of cases. Hence, further research is required to explore the application of biomarkers in evaluating the ROM of uncertain samples.

\section{Conclusions}

The application of biomarkers in distinguishing uncertain CNB samples of thyroid nodules is available and capable. CD56 negative or NGS positive suggests malignancy strongly for both FN and non-FN-lesions, which may be used as a "rule in" tool. The negativity of the integrated IHC and the integrated IHC-NGS implies a high possibility to be benign for non-FN-lesions and FN separately, which can work as a "rule out" tool. Considering the balance of specificity and sensitivity, NGS is the best for non-FNlesions and the integrated IHC-NGS is the best for FN.

Because NGS is not a universal technique, the working power of NGS depends largely on each laboratory's technical details. Pathologists should interpret NGS results based on the knowledge integrating the literature's reports and own lab's data. All of the data and analysis about NGS in our research are based on the specific commercial tool OncoAim ${ }^{\circledR}$.

Abbreviations CNB: Core needle biopsy; FNB: Fine-needle biopsy; H\&E: Hematoxylin and eosin; IHC: Immunohistochemistry; NGS: Next-generation sequencing; IHC-COMB: Immunohistochemistry combination; IHC-NGS: Immunohistochemistry and nextgeneration sequencing combination; PPV: Positive predictive value; NPV: Negative predictive value; NH: Nodular hyperplasia; FA: Follicular adenoma; FTC: Follicular thyroid carcinoma; PTC: Papillary thyroid carcinoma; FVPTC: Follicular variant of papillary thyroid carcinoma; CPTC: Conventional papillary thyroid carcinoma; FN: Follicular neoplasm; TCGA: The Cancer Genome Atlas; PDTC: Poorly differentiated thyroid carcinoma; ATC: Anaplastic thyroid carcinoma

Acknowledgements We thank Mr. Yang Li from the SingleCell Biotechnology Inc., Beijing, China, for his help in writing the NGS method section of the manuscript. We thank the technical support from the Singlera Genomics Inc., Shanghai, China, for NGS testing.

Author contribution YX initiated the study and wrote the manuscript. XL did gene testing. LL did the administration of the project and reviewed the slides. DL did IHC testing. LY collected data and reviewed slides. XL participated in project design and did the statistical analysis. JD collected and managed data. TL supervised the research. All authors have read and approved the final version of the manuscript.

Funding This work is supported by the Youth Clinical Research Project of Peking University First Hospital (No. 2019CR24).

Data availability The datasets used and analyzed during the current study are available from the corresponding author on reasonable request.

\section{Declarations}

Ethics approval and consent to participate The Ethics Committee of the Peking University First Hospital approves the use of all patient samples and clinical data and the informed consent exemption (ethical approval no.: (2018) Research No. 147).

Consent for publication Not applicable.

Competing interests The authors declare no competing interests.

Open Access This article is licensed under a Creative Commons Attribution 4.0 International License, which permits use, sharing, adaptation, distribution and reproduction in any medium or format, as long as you give appropriate credit to the original author(s) and the source, provide a link to the Creative Commons licence, and indicate if changes were made. The images or other third party material in this article are included in the article's Creative Commons licence, unless indicated otherwise in a credit line to the material. If material is not included in the article's Creative Commons licence and your intended use is not 
permitted by statutory regulation or exceeds the permitted use, you will need to obtain permission directly from the copyright holder. To view a copy of this licence, visit http://creativecommons.org/licenses/by/4.0/.

\section{References}

1. Teng WLY, Gao M, Huang G, Wu Y, Zhao J et al (2012) Guidelines on the diagnosis and treatment of thyroid nodules and differentiated thyroid carcinomas. Chin J Endocrinol Metab 28:779-797

2. Zhang M, Zhang Y, Fu S et al (2014) Thyroid nodules with suspicious ultrasound findings: the role of ultrasound-guided core needle biopsy. Clin Imaging 38:434-438

3. Lee HJ, Kim YJ, Han HY et al (2019) Ultrasound-guided needle biopsy of large thyroid nodules: core needle biopsy yields more reliable results than fine needle aspiration. J Clin Ultrasound 47:255-260

4. Jeong EJ, Chung SR, Baek JH et al (2018) A comparison of ultrasound-guided fine needle aspiration versus core needle biopsy for thyroid nodules: pain, tolerability, and complications. Endocrinol Metab (Seoul) 33:114-120

5. Wolinski K, Stangierski A, Ruchala M (2017) Comparison of diagnostic yield of core-needle and fine-needle aspiration biopsies of thyroid lesions: systematic review and meta-analysis. Eur Radiol 27:431-436

6. Xiong Y, Yan L, Nong L et al (2019) Pathological diagnosis of thyroid nodules based on core needle biopsies: comparative study between core needle biopsies and resected specimens in 578 cases. Diagn Pathol 14:10

7. Trimboli P, Nasrollah N, Guidobaldi L et al (2014) The use of core needle biopsy as first-line in diagnosis of thyroid nodules reduces false negative and inconclusive data reported by fine-needle aspiration. World J Surg Oncol 12:61

8. Jung CK, Baek JH, Na DG et al (2020) 2019 Practice guidelines for thyroid core needle biopsy: a report of the Clinical Practice Guidelines Development Committee of the Korean Thyroid Association. J Pathol Transl Med 54:64-86

9. Ricardo V. Lioyd RYO, Gunter Kloppel, Juan Rosai. WHO classification of tumours of endocrine organs. Lyon, France: International Agency for Research on Cancer (IARC),2017.

10. Dunderovic D, Lipkovski JM, Boricic I et al (2015) Defining the value of CD56, CK19, Galectin 3 and HBME-1 in diagnosis of follicular cell derived lesions of thyroid with systematic review of literature. Diagn Pathol 10:196

11. Saleh HA, Jin B, Barnwell J, Alzohaili O (2010) Utility of immunohistochemical markers in differentiating benign from malignant follicular-derived thyroid nodules. Diagn Pathol 5:9

12. Pyo JS, Kim DH, Yang J (2018) Diagnostic value of CD56 immunohistochemistry in thyroid lesions. Int J Biol Markers $33: 161-167$
13. Zargari N, Mokhtari M (2019) Evaluation of diagnostic utility of immunohistochemistry markers of TROP-2 and HBME-1 in the diagnosis of thyroid carcinoma. Eur Thyroid J 8:1-6

14. Song S, Kim H, Ahn SH (2019) Role of immunohistochemistry in fine needle aspiration and core needle biopsy of thyroid nodules. Clin Exp Otorhinolaryngol 12:224-230

15. Cancer Genome Atlas Research N. Integrated genomic characterization of papillary thyroid carcinoma. Cell 2014; 159: 676-690.

16. D'Cruz AK, Vaish R, Vaidya A et al (2018) Molecular markers in well-differentiated thyroid cancer. Eur Arch Otorhinolaryngol 275:1375-1384

17. Landa I, Ibrahimpasic T, Boucai L et al (2016) Genomic and transcriptomic hallmarks of poorly differentiated and anaplastic thyroid cancers. J Clin Invest 126:1052-1066

18. Acquaviva G, Visani M, Repaci A et al (2018) Molecular pathology of thyroid tumours of follicular cells: a review of genetic alterations and their clinicopathological relevance. Histopathology 72:6-31

19. Patel KN, Angell TE, Babiarz J et al (2018) Performance of a genomic sequencing classifier for the preoperative diagnosis of cytologically indeterminate thyroid nodules. JAMA Surg 153:817-824

20. Steward DL, Carty SE, Sippel RS et al (2019) Performance of a multigene genomic classifier in thyroid nodules with indeterminate cytology: a prospective blinded multicenter study. JAMA Oncol 5:204-212

21. Sadowski SM, Petrenko V, Meyer P et al (2019) Validation of molecular biomarkers for preoperative diagnostics of human papillary thyroid carcinoma in fine needle aspirates. Gland Surg 8:S62-S76

22. Choi SH, Baek JH, Lee JH et al (2015) Evaluation of the clinical usefulness of BRAFV600E mutation analysis of core-needle biopsy specimens in thyroid nodules with previous atypia of undetermined significance or follicular lesions of undetermined significance results. Thyroid 25:897-903

23. Choi SH, Baek JH, Lee JH et al (2016) Initial clinical experience with BRAF(V600E) mutation analysis of core-needle biopsy specimens from thyroid nodules. Clin Endocrinol (Oxf) 84:607-613

24. Crescenzi A, Trimboli P, Modica DC et al (2016) Preoperative assessment of TERT promoter mutation on thyroid core needle biopsies supports diagnosis of malignancy and addresses surgical strategy. Horm Metab Res 48:157-162

25. Jang EK, Kim WG, Kim EY et al (2016) Usefulness of NRAS codon 61 mutation analysis and core needle biopsy for the diagnosis of thyroid nodules previously diagnosed as atypia of undetermined significance. Endocrine 52:305-312

26. NCCN Clinical Practice Guidelines in Oncology. Thyroid carcinoma. In Version 3.2020-February 2 (ed). 2021; THYR2-4.

Publisher's note Springer Nature remains neutral with regard to jurisdictional claims in published maps and institutional affiliations. 